\title{
Acute abdomen in a mentally retarded patient: cecal volvulus
}

\author{
Ebubekir Gündeş ${ }^{1}$, Nedim Akgül ${ }^{2}$, Mustafa Mazıcan ${ }^{3}$, Ulaş Aday ${ }^{1}$, Durmuş Ali Çetin ${ }^{1}$, Hüseyin Çiyiltepe ${ }^{1}$ \\ ${ }^{1}$ Gastroenterological Surgery Department, Kartal Koşuyolu High Speciality and Training Hospital, Istanbul, Turkey \\ ${ }^{2}$ Department of General Surgery, Sarayönü State Hospital, Konya, Turkey \\ ${ }^{3}$ Department of Radiology, Ağrı State Hospital, Ağrı, Turkey
}

Gastroenterology Rev 2017; 12 (2): 159-161

DOI: https://doi.org/10.5114/pg.2017.68053

\begin{abstract}
Address for correspondence: Ebubekir Gündeş MD, Gastroenterological Surgery Department, Kartal Koşuyolu High Speciality and Training Hospital, Denizer cad. No: 22, 34000 Istanbul, Turkey, phone: +90 5058606740, e-mail: ebubekir82@hotmail.com
\end{abstract}

Cecal volvulus is a rare emergency surgical condition caused by the torsion of the cecum, distal ileum, and the proximal colon around the axial plane related to the lack of normal fixation of the cecum [1]. Cecal volvulus' clinical presentation varies a great deal, and while it can present with occasional abdominal pain it can also progress with severe abdominal pain. The severity of the pain depends on the level of cecal volvulus and the duration of intestinal obstruction [2]. It is quite hard to diagnose in the pre-operative period since it is very rare with no disease-specific symptoms [3]. The radiological method that is frequently used to confirm the diagnosis of cecal volvulus and to differentiate it from other acute emergency pathologies is computerised tomography (CT) $[4,5]$. Successful treatment in patients depends on early diagnosis and timely intervention [1, 3]. The most effective treatment method is emergency resection of the segment with torsion around its mesentery in order to prevent progression to necrosis, which increases the risk of morbidity and mortality [2].

This study discusses the case of a mentally retarded patient with acute abdomen related to cecal volvulus in light of the literature on the subject.

The 50-year-old male patient presented to the emergency with complaints of severe abdominal pain, vomiting, and inability to discharge gas-stool that had been going on for a day. His medical history revealed that he had mental retardation. It was seen that he had hypoactive intestinal sound upon listening with occasional metallic sounds during his abdominal examination. There was distinctive distension, widespread sensitivity, and rebound. The patient's biochemical parameters were within normal bounds and his white cell count was $16900 / \mathrm{mm}^{3}$. His stand ing direct abdominal graphy (X-ray) showed extreme- ly dilated colon segments with blurred haustrations in the shape of a reverse $U$, and air-fluid levels in the right side of the abdomen (Figure 1 ). His CT results demonstrated distension in the colonic loops beginning with the cecal level and complete cycle rotation (whirlpool sign) of intestinal loops, mesentery, and vascular structures in the lower right quadrant (Figure 2). We postulated that his condition might have been related to cecal torsion.

Emergency laparotomy was planned taking the patient's current physical examination, laboratory, and radiological results into consideration. The patient received fluid-electrolyte replacement and was taken into the operation. The abdomen was opened up through upper lower abdominal median incision during the procedure. The intra-abdominal exploration revealed that the cecum was torsioned and there were severely dilated, hyperaemic, and serosal lacerations. Meckel's diverticulum of about $7 \mathrm{~cm}$ in length was detected at about $40 \mathrm{~cm}$ to the terminal ileum (Figure 3). The patient had right hemicolectomy, small bowel resection including Meckel's diverticulum, and primary anastomosis.

Colonic volvulus is the third most frequent cause of colon obstruction in developed countries following cancer and diverticular disease [1]. Cecal volvulus is an emergency surgical condition caused by the torsion of the cecum, distal ileum, and proximal colon around the axial plane related to the lack of normal fixation of the cecum $[1,2]$. Cecal volvulus is the result of abnormal mobility related to the inappropriate union of cecal mesentery with parietal periton in the back [6]. Chronic constipation, abdominal masses, late pregnancy, previous history of abdominal surgery, extended immobilisation, paralytic ileus, and colonoscopy are suggested to be among the predisposing factors and aetiology of 


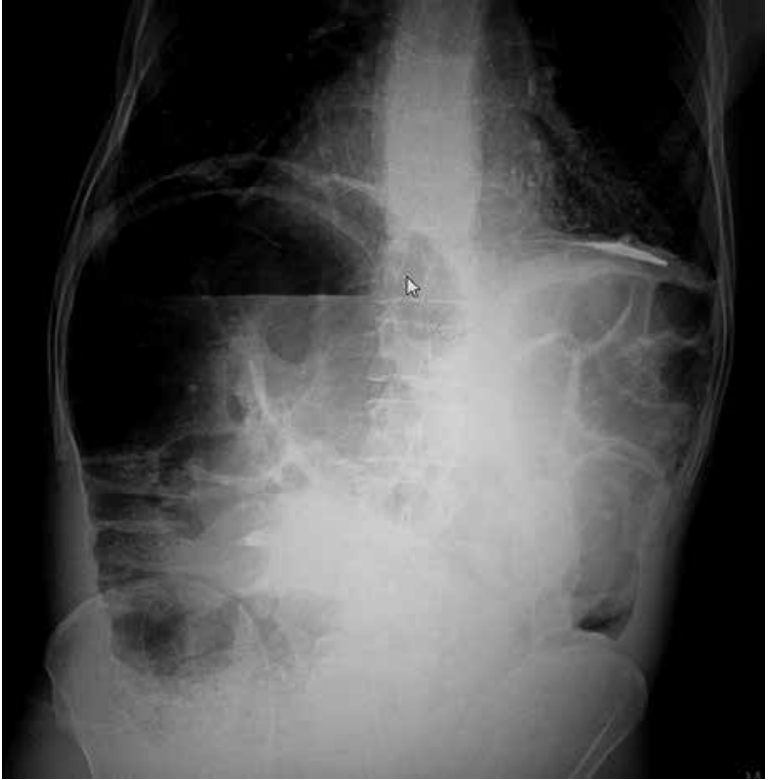

Figure 1. Air-fluid level at the colonic level and reverse $U$ outlook as revealed by standing direct abdominal graphy (X-ray)

cecal volvulus [2]. The most common symptoms seen in patients with cecal volvulus are abdominal pain accompanied by nausea, vomiting, and abdominal distension [2]. If intestinal vitality is disturbed, peritoneal irritation signs are added to the picture [7]. Symptoms and signs that would suggest colonic obstruction were present in our patient.

Radiological findings that can be detected by direct graphies (X-ray) in cecal volvulus are cecal dilatation, air-fluid levels, small bowel dilatation, lack of gas in the

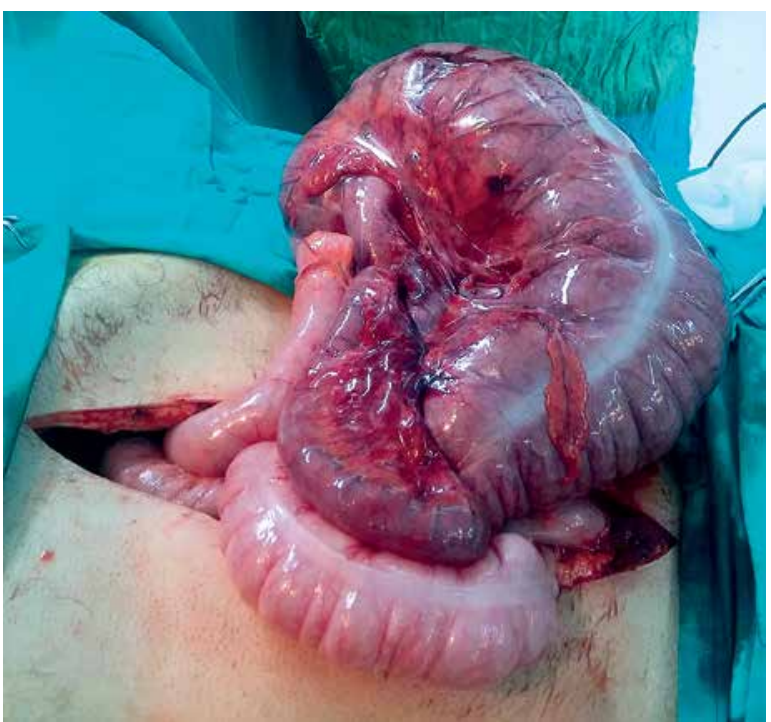

Figure 3. View from the surgical procedure

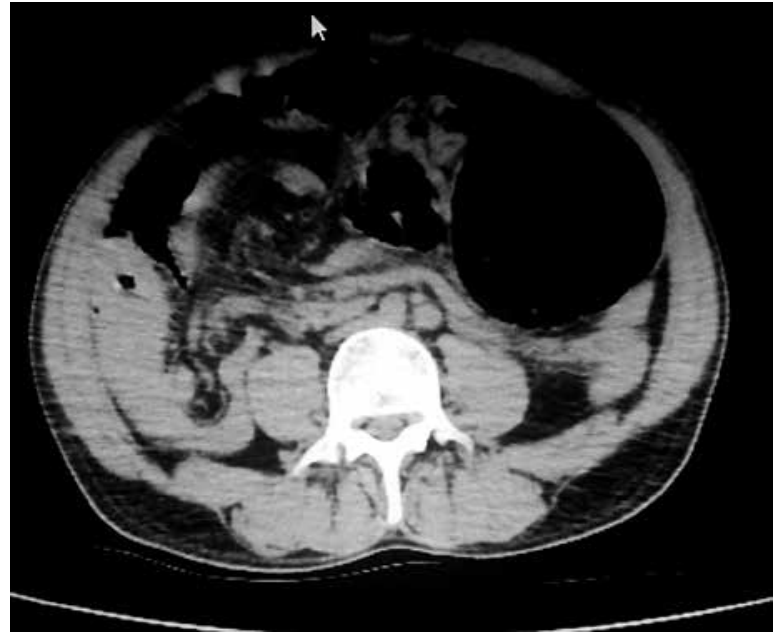

Figure 2. Whirlpool sign as shown by computerised tomography

distal colon, and the presence of dilated small bowel loops next to the dilated cecum [4]. Because CT is a more common imaging method in patients with acute abdominal pain, familiarisation with cecal volvulus signs will prevent misdiagnoses and delays in surgical intervention. Bird-beak sign refers to the pointed convergent outlook of the obstructed loops at the torsion point [5]. Whirlpool sign, on the other hand, is defined as the outlook caused by the intertwined cecal and distal ileum loops and dilated veins, and it has been suggested that the tightness of the whirlpool points to the level of cecal rotation [4, 5]. Our patient's standing direct abdominal graph also showed reverse $U$ sign and air-fluid levels while his CT showed whirlpool sign at the colonic level.

Resection is necessary in the case of strangulation and perforation, and if the cecum wall is very thin resection is also recommended. The success rate of detorsion by colonoscopy is very low (12.5\%). Resection, cecopexy, cecostomy, and cecopexy with cecostomy can be used as surgical treatment. The recurrence rate following resection is distinctively low [7]. Our patient received right hemicolectomy and primary anastomosis because he had necroses and serosal perforations in a wide area.

The formation of cecal volvulus depends on multifactorial reasons in the presence of cecal mobility abnormality. Although cecal volvulus is a rare type of colonic volvulus, it should be taken into consideration in the differential diagnosis of patients presenting with colonic obstruction symptoms. Successful treatment of patients with colonic volvulus depends on early diagnosis and timely intervention. The preferred method of surgical procedure should be designated by considering the general condition of the patient, intra-abdominal 
contamination, and the ischaemic condition in colonic segments. If the patient is in a good condition resection-primary anastomosis should be the preferred method of treatment.

\section{Conflict of interest}

The authors declare no conflict of interest.

\section{References}

1. Hashimoto Y, Shigemoto S, Nakashima A, et al. Successful preoperative diagnosis of rare bowl obstruction: cecal volvulus. J Gastrointest Surg 2008; 12: 202-4.

2. Mediba TE, Thomson SR. The management of cecal volvulus. Dis Colon Rectum 2002; 45: 264-7.

3. Pahlman L, Enblad P, Rudber C, et al. Volvulus of the colon. A review of 93 cases and current aspect of treatment. Acta Chir Scand 1989; 155: 53-6.

4. Rosenblat JM, Rozenblit AM, Wolf EL, et al. Findings of cecal volvulus at C.T. Radiology 2010; 256: 169-75.

5. Moore CJ, Cori FM, Fishman EK. CT of cecal volvulus: unravelinf the image. AJR Am J Roentgenol 2001; 177: 95-8.

6. Rogers RL, Harford FJ. Mobile cecum syndrome. Dis Colon Rectum 1984; 27: 399-402.

7. Habre J, Sautot-Vial N, Marcotte C, et al. Caecal volvulus. Am J Surg 2008; 196: 48-9.

Received: 22.07.2016

Accepted: 17.08 .2016 